\title{
Risks of loosening of a prosthetic glenoid implanted in retroversion
}

\author{
Alain Farron, MD, ${ }^{a}$ Alexandre Terrier, $\mathrm{PhD},{ }^{\mathrm{b}}$ and Philippe Büchler, $\mathrm{PhD},{ }^{\mathrm{b}}$ Lausanne, Switzerland
}

Osteoarthritis of the shoulder is frequently associated with posterior glenoid wear, which may be difficult to correct during shoulder arthroplasty. This study was designed to evaluate the risks that a prosthetic glenoid implanted in retroversion will loosen. The scapula, the humerus, the rotator cuff, and a total shoulder prosthesis were reconstructed with a 3-dimensional finite element model. The glenoid was placed in 5 different angles of retroversion $10^{\circ}, 5^{\circ}, 10^{\circ}, 15^{\circ}$, and $20^{\circ}$. Location of the glenohumeral contact point, articular pressure, bone and cement stress, and micromotion around the glenoid implant were calculated during internal and external rotation. Glenoid retroversion induced a posterior displacement of the glenohumeral contact point during internal and external rotation, inducing a significant increase of stress within the cement mantel $(+326 \%)$ and within the glenoid bone $(+162 \%)$. Furthermore, a major increase of micromotion was measured at the bone-cement interface (+706\%). According to this study, glenoid retroversion exceeding $10^{\circ}$ should be corrected during total shoulder arthroplasty. If the correction is impossible, not replacing the glenoid should be considered. (J Shoulder Elbow Surg 2006; 15:52 1-526.)

steoarthritis of the shoulder is frequently associated with posterior glenoid wear. 6, 15,23 The reasons remain unclear, but are probably multifactorial. The excessive stiffness of the anterior soft tissues after previous surgical procedures performed through an anterior approach is classically recognized as a cause of posterior glenoid erosion. $1,7,13$ Posterior glenoid wear is also frequently seen without any previous surgery, however. In recent years, primary glenoid dysplasia, associated with increased glenoid retroversion and sometimes with a

From the a Orthopaedic Hospital, University of Lausanne and bthe Orthopaedic Research Laboratory, Swiss Federal Institute of Technology

Reprint requests: Alain Farron, Hôpital Orthopédique, Av. Pierre Decker 4, 1005 Lausanne, Switzerland (E-mail: alain.farron@ chuv.ch).

Copyright ( 2006 by Journal of Shoulder and Elbow Surgery Board of Trustees.

$1058-2746 / 2006 / \$ 32.00$

doi: 10.1016/i.jse.2005.10.003 static posterior subluxation, has also been evoked as a cause of shoulder osteoarthritis. ${ }^{24,25}$ Among the other possible causes, the effects of muscular imbalance (eg, in association with neurologic problems) and the influence of the humeral side of the joint (especially retroversion of the humeral head) are less frequently reported.

During shoulder arthroplasty, correction of a retroverted or posteriorly eroded glenoid is a difficult task. Reaming of the anterior part of the glenoid ${ }^{14}$ may significantly reduce bone stock, leading to concerns about the stability of the prosthetic component. Furthermore, this procedure will medially displace the center of rotation, which may provoke impingement between the coracoid process and the humeral head. Bone grafting of the posterior glenoid, , 16,19 associated or not with an osteotomy, is an alternative. This option is often technically difficult, making the procedure significantly more complex; furthermore, the results are also unpredictable. Consequently, glenoid retroversion is sometimes not, or only partially, corrected during total shoulder replacement.

Malposition of the prosthetic components has been reported as a cause of unsatisfactory results after total shoulder arthroplasty. ${ }^{8}$ Experimental and numeric studies have shown that misalignment of the glenoid may lead to asymmetric load of the component and cement failure. ${ }^{10,17,18}$ The specific consequences of posterior glenoid retroversion on the survival of the implant are still not well known. Particularly, the effects on micromotion at the bone-cement interface and the stress transmitted to the underlying bone have not yet been studied. The objective of this work was, therefore, to analyze, by the mean of a finite element model, the biomechanics and the risks of loosening of a glenoid implanted with different angles of retroversion. The amount of retroversion to correct was also evaluated.

\section{MATERIALS AND METHODS}

\section{Shoulder model}

A 3-dimensional finite element model of the shoulder was developed ${ }^{2,3}$ and specifically adapted for this study. Data were obtained from an intact cadaver shoulder without any macroscopic or radiologic signs of pathology. Computed tomography sequences allowed reconstruction of bone shapes and density, 

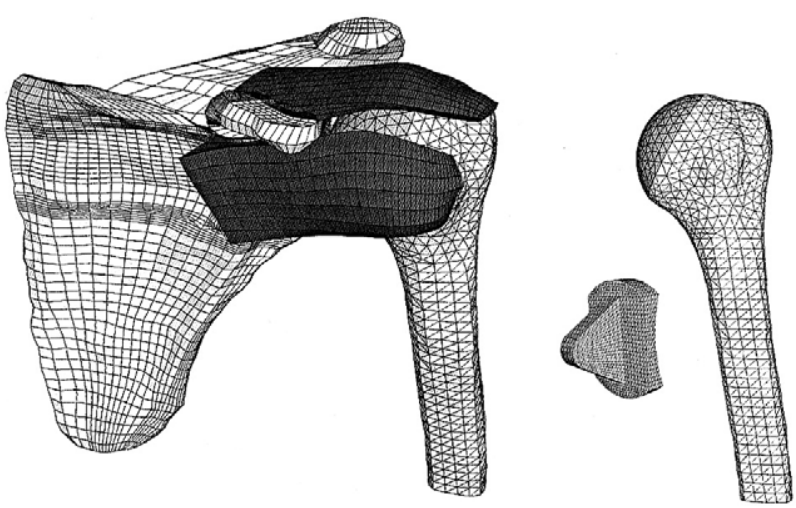

Figure 1 Finite element model of the shoulder, including the total prosthesis.

and accurate dissection showed the precise location of the muscle insertions. This computer model included the proximal half of the humerus, the entire scapula, and the 3 major rotator cuff muscles (infraspinatus, supraspinatus, and subscapularis).

A total shoulder prosthesis was numerically implanted into the model, according to the surgeon's indications (Figure 1). The metallic prosthetic humeral head was a portion of a sphere (length, $46 \mathrm{~mm}$; height, $17 \mathrm{~mm}$; radius of curvature, $24 \mathrm{~mm}$ ), reconstructing anatomically the proximal humerus, as implants of the third generation do. The glenoid component, made of polyethylene, was keeled and had a flat back. The articular surface was spherical (radius of curvature, $34 \mathrm{~mm}$ ); therefore, the radial mismatch between the humeral head and the glenoid was 10 $\mathrm{mm}$. The center of the prosthetic glenoid was placed at the center of the natural glenoid. In the reference position $\left(0^{\circ}\right.$ of retroversion), orientation of the prosthetic and natural glenoids was identical. The same implant was then numerically placed in 4 other positions of retroversion: $5^{\circ}, 10^{\circ}, 15^{\circ}$, and $20^{\circ}$. When placed in $15^{\circ}$ or $20^{\circ}$ of retroversion, the extremity of the keel perforated the anterior bony cortex. A $1-\mathrm{mm}$ polymethylmethacrylate cement layer was placed between glenoid and bone.

In this study, the humerus was considered as a rigid body, whereas an inhomogeneous elastic law described the scapula, taking into account the bone density distribution. ${ }^{20}$ An incompressible, hyperelastic exponential law characterized the mechanical behavior of the muscles. The implant-cement interface was bonded, whereas micromotion was allowed at the cement-bone interface.

\section{Loading conditions}

The neutral position of the glenohumeral joint $10^{\circ}$ of rotation) was defined as the position in which the center of the humeral articular surface faced the cen- ter of the glenoid fossa. A progressive displacement of the scapular extremity of the subscapularis (respectively, the infraspinatus) was imposed to generate $60^{\circ}$ of internal rotation (respectively, $30^{\circ}$ of external rotation). These displacements, which represented the muscles' contraction, resulted in a maximal force of $54 \mathrm{~N}$ in the subscapularis and $42 \mathrm{~N}$ in the infraspinatus at $60^{\circ}$ of internal rotation, and $16 \mathrm{~N}$ in the subscapularis and $21 \mathrm{~N}$ in the infraspinatus at $30^{\circ}$ of external rotation. The supraspinatus, which sustained a constant force of $1 \mathrm{~N}$, acted as a stabilizer in the inferior and superior directions. Boundary conditions imposed on the distal part of the humerus were chosen to allow movements of flexion, extension, abduction, or adduction.

The effects of prosthetic glenoid retroversion on the risks of implant loosening and failure were analyzed through different mechanical variables: the glenohumeral contact point and pressure, the cement stress, the relative micromotion at the cement-bone interface, and the osseous glenoid stress. These values were calculated throughout the full range of external and internal rotation.

\section{RESULTS}

\section{Glenohumeral contact point and location}

Glenoid retroversion influenced the location of the contact point (Figure 2), which moved posteriorly with increasing angles of retroversion in internal as well as in external rotation. On the other hand, retroversion had only a moderate effect on the maximal contact pressure, which slightly decreased $(-16 \%)$. Without retroversion, peaks of stress within the glenoid component were symmetric and mainly located in the center of the implant. As retroversion increased to $20^{\circ}$, peaks of stress moved to the posterior aspect of the glenoid component.

\section{Cement stress}

The glenoid retroversion increased the peaks of cement stress (Figure 3 ) up to $326 \%$ at $20^{\circ}$ of retroversion. The maximum principal stress reached 9.4 $\mathrm{MPa}$ at $60^{\circ}$ of internal rotation and for $20^{\circ}$ of retroversion. Furthermore, the implant orientation had an effect on the stress distribution. Without retroversion, peaks of stress were symmetrically located at the anterior and posterior edges between the keel and the glenoid back. With increasing angles of retroversion, peaks of stress developed mainly in the posterior part of the cement mantel.

\section{Micromotion}

Micromotion (Figure 4) generated at the bonecement interface was strongly influenced by glenoid retroversion. Above $10^{\circ}$ of retroversion, there was an 


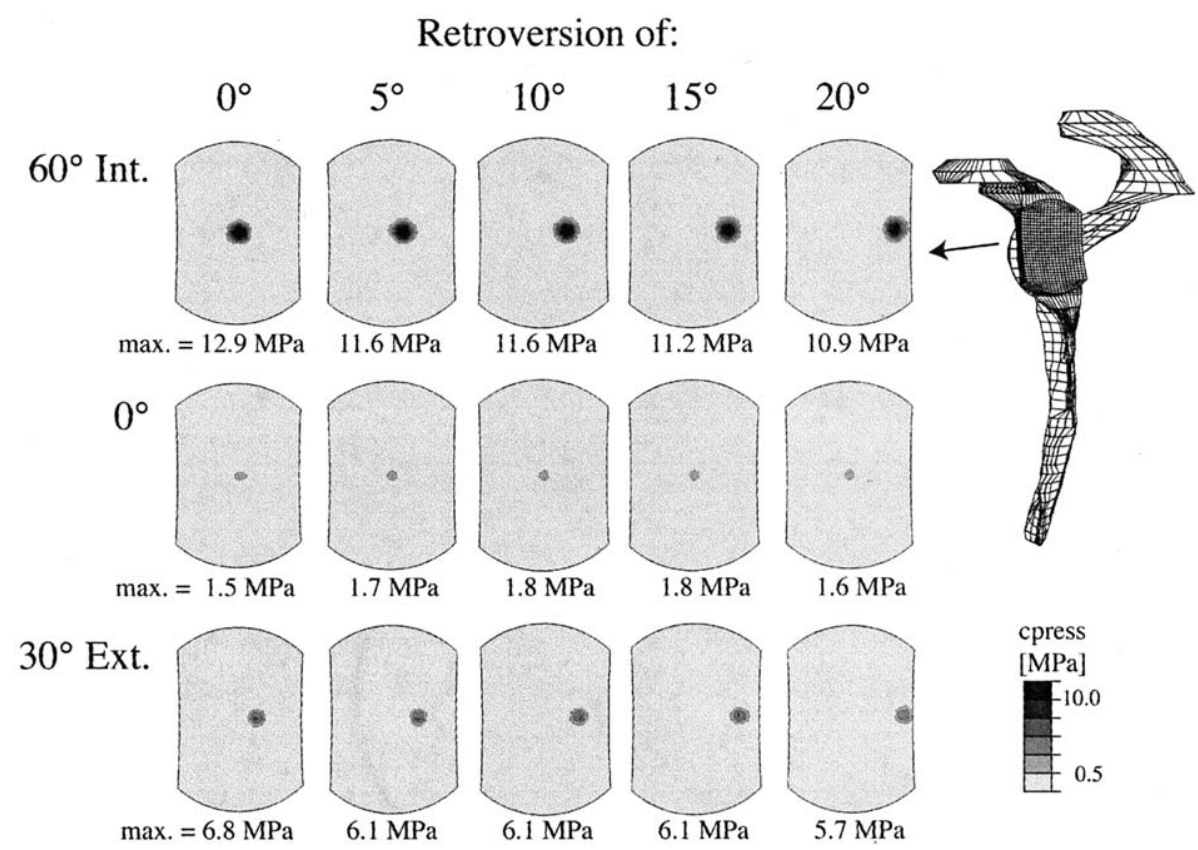

Figure 2 Contact pressures of the humeral head on the glenoid surface at $60^{\circ}$ of internal rotation (top), $0^{\circ}$ (middle), and $30^{\circ}$ of external (bottom) rotation for the 5 angles of glenoid retroversion.

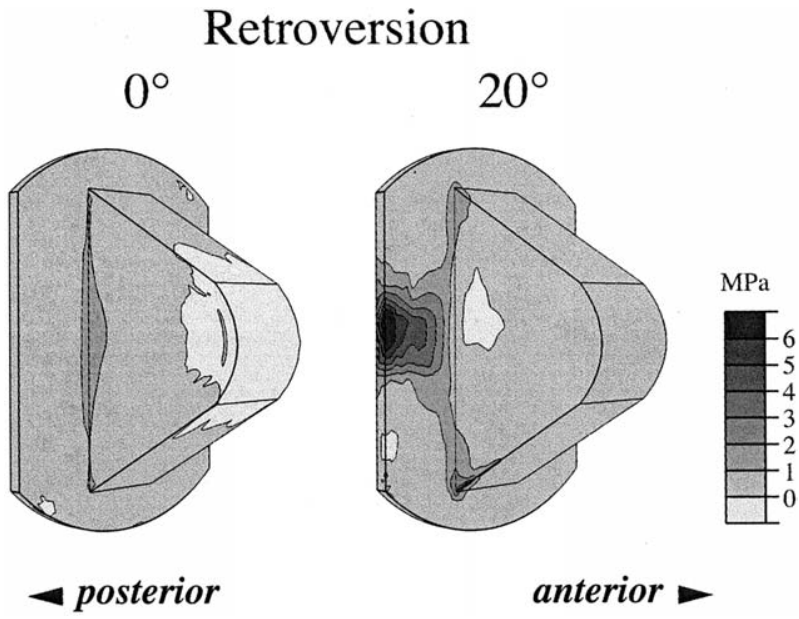

Figure 3 Maximal stresses (maximal principal stress) generated in the cement layer at $0^{\circ}$ and $20^{\circ}$ of retroversion (at $60^{\circ}$ of internal rotation).

exponential increase of maximal $(+706 \%)$ and mean $(+669 \%)$ micromotion for external and internal rotations. Without retroversion, micromotion was distributed all around the bone-cement interface. At $20^{\circ}$ of retroversion, however, peaks of tangential micromotion (slipping) were mainly located at the anterior side of the keel and perpendicular micromotion (debonding) was located under the anterior back of the glenoid. These above observations were similar for internal or external rotation.

\section{Stress in glenoid bone}

Retroversion of the implant had also an effect on the stress developed within the glenoid bone (Figure 5). Increasing angles of retroversion induced larger Von Mises stress $(+162 \%)$, mainly in the posterior aspect of the glenoid. Furthermore, the volume submitted to high stresses was significantly larger.

\section{DISCUSSION}

Glenoid loosening is still a matter of concern and may preclude good results after total shoulder replacement. Loading conditions of the glenoid influence the stresses developed within and around the components and, consequently, the survival of the implants. Because loading conditions depend on the implant orientation, posterior erosion of the glenoid due to shoulder osteoarthritis could be an important feature associated with glenoid loosening, if not corrected. Accordingly, this study was designed to evalvate the consequences of glenoid orientation on shoulder biomechanics.

Our results showed that all the mechanical variables relevant for the mechanisms of loosening were negatively influenced by the glenoid retroversion. The stress within cement and bone as well as the micromotion around the implants increased significantly with retroversion, especially for angles of more than $10^{\circ}$. When the articular surface of the glenoid was no longer perpendicular to the axis of the rotator cuff, as 
$60^{\circ}$ Internal Rotation
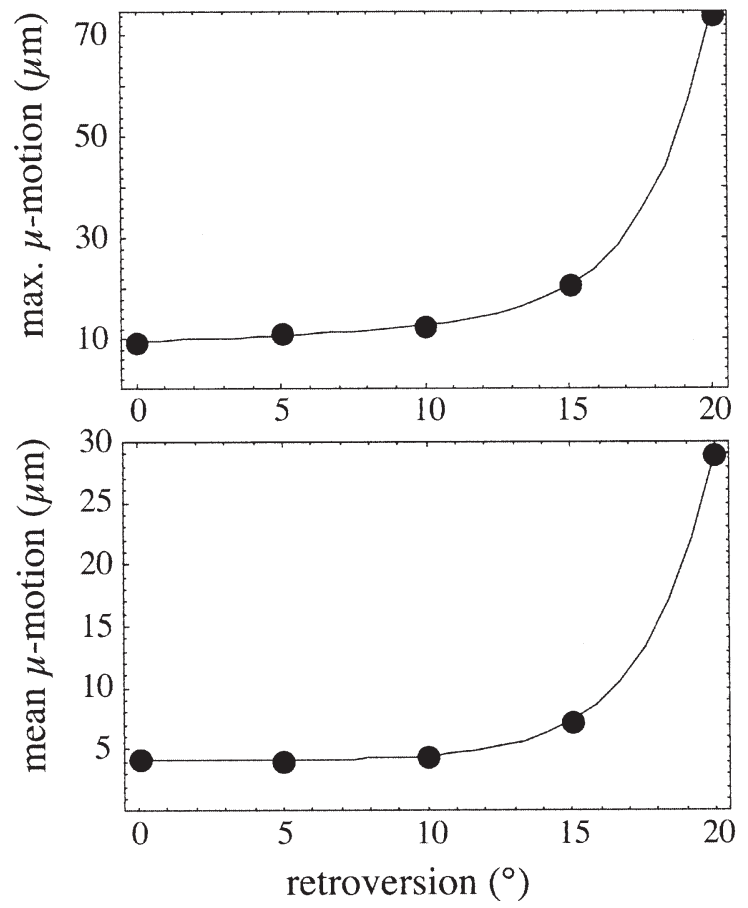

$30^{\circ}$ External Rotation
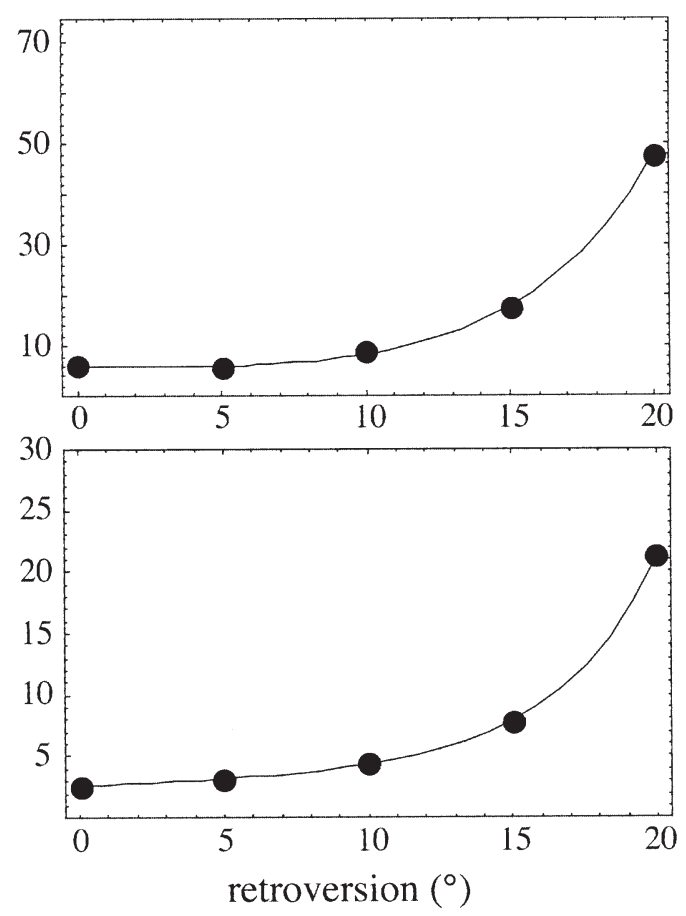

Figure 4 Maximal (top) and mean (bottom) tangential (slipping) micromotion at the cement-bone interface as a function of the glenoid retroversion at $60^{\circ}$ of internal (left) and $30^{\circ}$ of external (right) rotation.

happens in retroversion, the net glenoid reaction force had a posterior component (Figure 6). This posterior force displaced the contact point between the humeral head and the glenoid posteriorly, creating an asymmetric load on the posterior glenoid. The posterior force was present in external as well as in internal rotation. Consequently, as this study has clearly demonstrated, the deleterious effect of glenoid retroversion will be expressed in internal as well as in external rotation.

The posterior load on the glenoid generated an increase of stress and micromotion. Because the mean and the maximal micromotion follow the same trend, the increase should not be considered as a local phenomenon. At $20^{\circ}$ of retroversion, the distribution of micromotion showed a peak of perpendicular micromotion (debonding) under the anterior back of the glenoid component and a peak of tangential micromotion (slipping) along the anterior part of the keel. This fact is clearly a consequence of the wellknown rocking-horse effect. It is, therefore, the most probable cause of the biomechanical changes observed in our study, which leads to concern about the long-term survival of the implant. The loosening of the implant is a progressive phenomenon, however, which the present numeric model did not take into account. To analyze the time progression of the loos- ening process, a bone remodeling law ${ }^{4}$ should be included with the finite element model.

Clinical studies have emphasized the importance of glenoid retroversion or wear on the results after shoulder arthroplasty. Levine et al ${ }^{12}$ found that prosthetic shoulder replacement for osteoarthritis gave less satisfactory results if the glenoid had posterior wear ${ }^{12}$; however, the study was limited to hemiarthroplasties. lannotti and Norris ${ }^{11}$ examined the influence of preoperative factors on the outcome of shoulder arthroplasty for osteoarthritis. They found that, in the case of preoperative posterior glenoid wear, the outcome was better after total shoulder replacement than after hemiarthroplasty. They did not analyze the postoperative radiographs, however, and were thus unable to correlate the results with the correction of the posterior wear. They also reported worse results in cases of posterior humeral head subluxation without mentioning if the situation had been addressed during the surgical procedure.

The importance of component positioning during shoulder arthroplasty is now recognized and has recently led to many studies. In a clinical review of unsatisfactory results after total shoulder arthroplasty, Hasan et $\mathrm{al}^{8}$ found that malposition of the components was present in $28 \%$ of the cases. However, they did not mention if the glenoids or the humeral stems were 

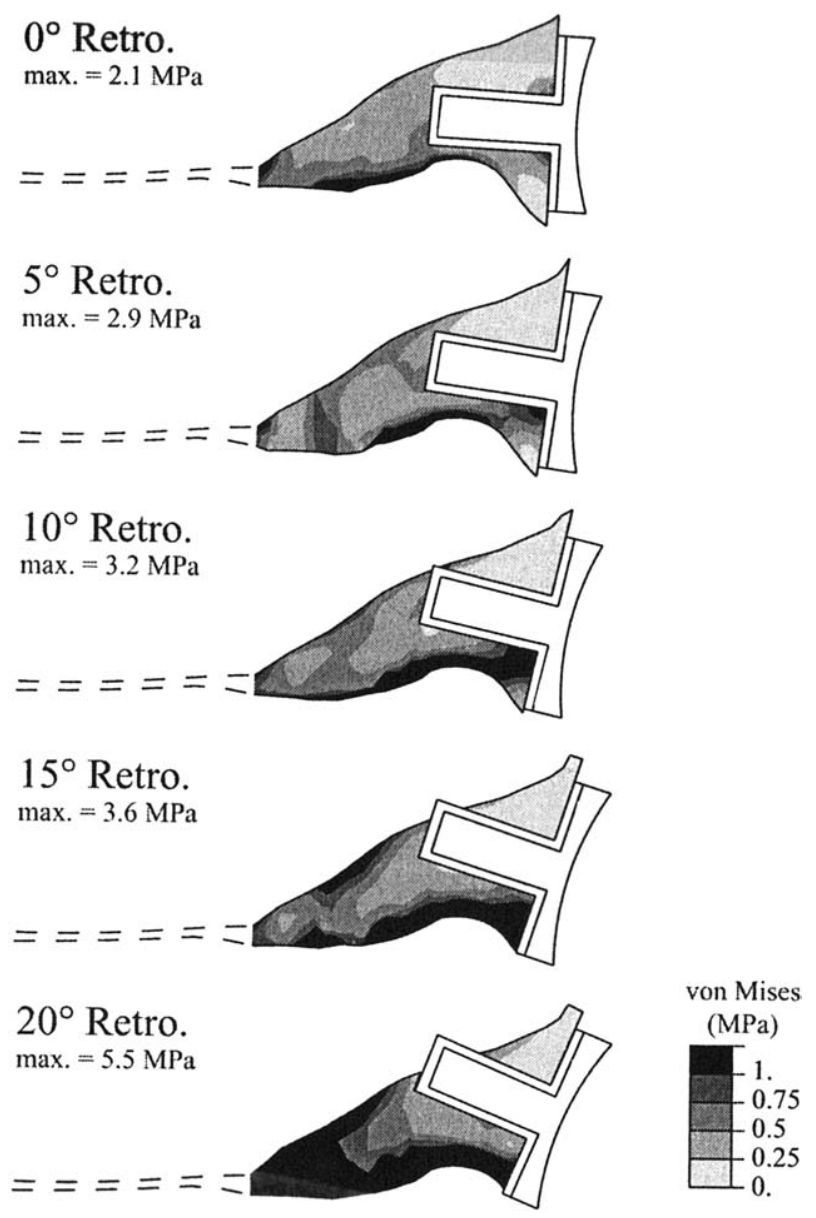

Figure 5 Glenoid intraosseous stresses (Von Mises) at $60^{\circ}$ of internal rotation for the 5 different retroversions.

not placed correctly. Oosterom et al ${ }^{18}$ showed in an experimental study that superior inclination of the glenoid might lead to cranial displacement of the humeral head and asymmetric loading of the glenoid during abduction but did not analyze the effects of component malposition in the axial plane. In a cadaver study, Nyffeler et al ${ }^{17}$ found that glenoid version provoked a displacement of the glenohumeral contact point, inducing a tilting moment on the glenoid during rotations. Hopkins et al, ${ }^{10}$ in a numeric study, analyzed the effects of glenoid alignment on the cement mantel during abduction and found that misalignment, especially in superoinferior position, might lead to failure of the cement mantel. A similar conclusion can be derived from the present work because cement stress exceeded its fatigue limit $^{5}$ for $20^{\circ}$ of retroversion; therefore, the stress generated within the cement is an important factor for implant stability. Cement thickness, which also influences cement stress distribution, was not specifically evaluated in this study; however, the use of a $1-\mathrm{mm}$ cement thickness seems to be favorable. ${ }^{21}$
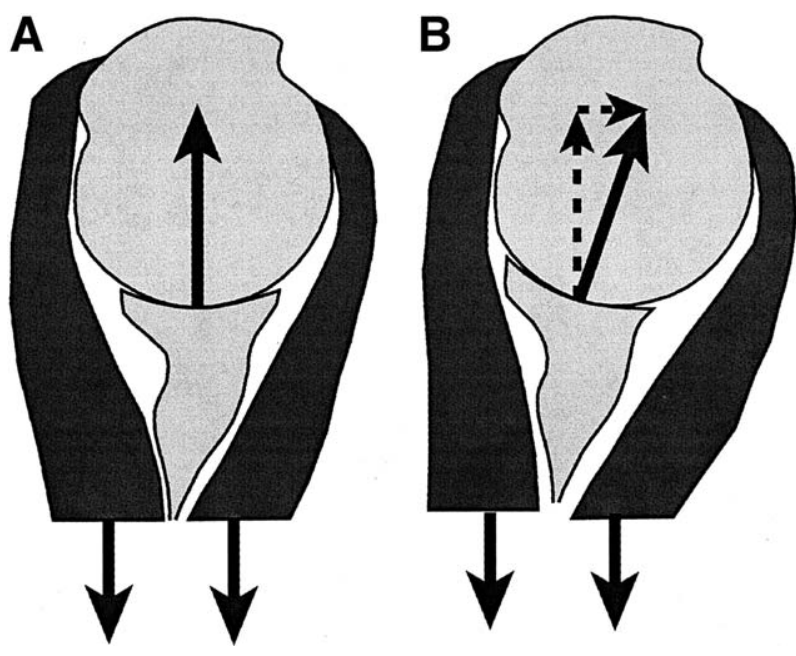

Figure 6 Effects of the retroversion on the net reaction force exerted by the glenoid on the humeral head. Without any retroversion (A) the net reaction force is perpendicular to the glenoid. In the retroverted case (B), the net reaction force has a posterior component.

Maximal and mean micromotion increased exponentially when the retroversion angle exceeded $10^{\circ}$ for internal or external rotation. Maximal micromotion $(74.2 \mu \mathrm{m})$ led to concerns about long-term stability of the prosthesis. A strict correlation between distribution/occurrence of micromotion and clinical observations (radiolucent lines) was not possible because there is no clinical study of the specific location of radiolucent lines related to the orientation of the prosthetic glenoid. Walch et al, ${ }^{26}$ in a clinical multicentric study, reported the occurrence of radiolucent lines around the prosthetic glenoid. However, they correlated their results to the mismatch between the humeral head and the glenoid but not to the glenoid retroversion, although a significant number of patients had preoperatively posterior glenoid erosion. The present numeric model was used in another study ${ }^{22}$ that also evaluated the effect of the articular mismatch on micromotion at the bone-cement interface. Our main conclusion was that retroversion of the glenoid had a more significant effect than the mismatch.

The main limitation of this study is the movements simulated: only rotations were analyzed. However, although abduction forces are usually preferred to model typical glenohumeral joint loading, pure rotations were more relevant for analyzing the consequences of glenoid retroversion because they occur in the same plane as the implant misalignment. The present study was therefore deliberately limited to a typical specific clinical situation, glenoid retroversion, which is frequently associated with glenohumeral osteoarthritis.

Another limitation is that the numeric results were not validated against direct experimental measure- 
ments. However, it is commonly accepted that, providing the different material properties are correctly described, finite elements technique may be used without any further experimental assessments to calculate the motion and stress state of deformable structures. Furthermore, it should be noted that the present model has also been applied to analyze other biomechanical aspects of the shoulder pathology and provided results that were in agreement with clinical observations.

\section{CLINICAL RELEVANCE}

This work highlights the importance of glenoid component retroversion after shoulder arthroplasty; above $10^{\circ}$ of retroversion, major biomechanical alterations were observed that could significantly increase the risks of glenoid loosening. According to this study, retroversion measured with computed tomography before surgery should be corrected to remain below $10^{\circ}$. If correction is impossible, not replacing the glenoid should be considered.

\section{REFERENCES}

1. Bigliani LU, Weinstein DM, Glasgow MT, Pollock RG, Flatow EL. Glenohumeral arthroplasty for arthritis after instability surgery. J Shoulder Elbow Surg 1995;4:87-94.

2. Buchler P, Rakotomanana L, Farron A. Virtual power based algorithm for decoupling large motions from infinitesimal strains: application to shoulder joint biomechanics. Comput Methods Biomech Biomed Engin 2002;5:387-96

3. Buchler $P$, Ramaniraka NA, Rakotomanana LR, lannotti JP, Farron A. A finite element model of the shoulder: application to the comparison of normal and osteoarthritic joints. Clin Biomech 2002; 17:630-9

4. Buchler P, Pioletti DP, Rakotomanana LR. Biphasic constitutive laws for biological interface evolution. Biomech Model Mechanobiol 2003: 1:239-49

5. Davies JP, Burke DW, O'Connor DO, Harris WH. Comparison of the fatigue characteristics of centrifuged and uncentrifuged Simplex P bone cement. J Orthop Res 1987;5:366-71 .

6. Friedman RJ, Hawthorne KB, Genez BM. The use of computerized tomography in the measurement of glenoid version. J Bone Joint Surg Am 1992 74: 1032-7.

7. Green A, Norris TR. Shoulder arthroplasty for advanced glenohumeral arthritis after anterior instability repair. J Shoulder Elbow Surg. 2001;10:539-45

8. Hasan SS, Leith JM, Campell B, Kapil R, Smith KL, Matsen FA 3rd. Characteristics of unsatisfactory shoulder arthroplasties. J Shoulder Elbow Surg 2002;1 1:431-41.

9. Hill JM, Norris TR. Long-term results of total shoulder arthroplasty following bone-grafting of the glenoid. J Bone Joint Surg Am 2001:83:877-83.

10. Hopkins AR, Hansen UH, Amis AA, Emery R. The effects of glenoid component alignment variations on cement mantle thickness stresses in total shoulder arthroplasty. J Shoulder Elbow Surgery 2004; 13:668-675

1 1. lannotti JP, Norris TR. Influence of preoperative factors on outcome of shoulder arthroplasty for glenohumeral osteoarthritis. J Bone Joint Surg Am 2003;85:25 1-8.

12. Levine WN, Djurasovic M, Glasson JM, Pollock RG, Flatow EL, Bigliani LU. Hemiarthroplasty for glenohumeral osteoarthritis: results correlated to degree of glenoid wear. J Shoulder Elbow Surg 1997;6:449-54

13. Matsen FA, Lippitt SB, Sidles JA, Harrymann DT. Shoulder motion. In: Practical evaluation and management of the shoulder. Philadelphia: W.B. Saunders Company; 1994. p. 39-40.

14. Matsen FA, Lippitt SB, Sidles JA, Harrymann DT. Smoothness. In: Practical evaluation and management of the shoulder. W.B. Saunders Company; 1994. p. 211.

15. Mullaji AB, Beddow FH, Lamb GH. CT measurement of glenoid erosion in arthritis. J Bone Joint Surg 1994;76:384-8.

16. Neer CS, Morrison DS. Glenoid bone-grafting in total shoulder arthroplasty. J Bone Joint Surg Am 1988;70: 1 154-62.

17. Nyffeler WR, Sheikh R, Jakob HAC, Gerber C. Influence of version on loading pattern of glenoid components: an experimental investigation. J Shoulder Elbow Surg 2000;9:549.

18. Oosterom R, Rozing PM, Bersee HEN. Effect of glenoid component inclination on its fixation and humeral head subluxation in total shoulder arthroplasty. Clin Biomech 2004; 19: 1000-8.

19. Steinmann SP, Cofield RH. Bone grafting for glenoid deficiency in total shoulder replacement. J Shoulder Elbow Surg 2000;9: 361-7.

20. Terrier A. Adaptation of bone to mechanical stress: theoretical model, experimental identification and orthopaedic applications. PhD thesis. EPFL, Lausanne, 1999.

21. Terrier A, Buchler P, Farron A. Bone-cement interface of the glenoid component: stress analyzing for varying cement thickness. Clinic Biomech 2005;20:710-17

22. Terrier A, Buchler P, Farron A. Glenoid stability after shoulder arthroplasty: influence of glenohumeral conformity. J Shoulder Elbow Surg 2006 (in press).

23. Walch G, Boulahia A, Boileau P, Kempf JF. Primary glenohumeral osteoarthritis: clinical and radiographic classification. Acta Orthopaedica Belgica 1998;64 Suppl 2:46-52.

24. Walch G, Badet R, Boulahia A, Khoury A. Morphologic study of the glenoid in primary glenohumeral osteoarthritis. J Arthroplasty 1999; 14:756-60.

25. Walch G, Ascani C, Boulahia A, Nove-Josserand L, Edwards TB. Static posterior subluxation of the humeral head: an unrecognized entity responsible for glenohumeral osteoarthritis in the young adult. J Shoulder Elbow Surg 2002; 11 :309-14.

26. Walch G, Edwards TB, Boulahia A, Boileau P, Mole D, Adeleine $P$. The influence of glenohumeral prosthetic mismatch on glenoid radio lucent lines: results of a multicenter study. J Bone Joint Surgery Am 2002;84:21 86-91 . 